Draft VERSION MARCh 6, 2019

Typeset using IATEX RNAAS style in AASTeX62

\title{
The double Blue Straggler Sequence in NGC 2173: Yes, A Field Contamination ARTEFACT!

\author{
Emanuele Dalessandro, ${ }^{1}$ Francesco R. Ferraro, ${ }^{2}$ Nate Bastian, ${ }^{3}$ Mario Cadelano, ${ }^{2}$ Barbara Lanzoni, ${ }^{2}$ and Silvia Raso ${ }^{2}$ \\ ${ }^{1}$ INAF - Astrophysics and Space Science Observatory Bologna, Via Gobetti 93/3 I-40129 Bologna, Italy \\ ${ }^{2}$ Universitá di Bologna, Dipartimento di Fisica e Astronomia, Via Gobetti 93/2 I-40129 Bologna, Italy
} \\ ${ }^{3}$ Astrophysics Research Institute, Liverpool John Moores University, 146 Brownlow Hill, Liverpool, L3 5RF, UK
}

(Received February 13, 2019; Accepted February 18, 2019)

Submitted to RNAAS

Keywords: blue stragglers; galaxies: star clusters: individual (NGC2173); Hertzsprung-Russel and $\mathrm{C}-\mathrm{M}$ diagrams

\section{INTRODUCTION}

Li et al. (2018a, hereafter L18a) claimed that the young stellar cluster NGC 2173 in the Large Magellanic Cloud (LMC) harbours a bifurcated sequence of blue straggler stars (BSSs), similar to those detected in a few dynamically old globular clusters (Ferraro et al. 2009; Dalessandro et al. 2013; Simunovic et al. 2014). However, Dalessandro et al. (2019, hereafter D19) re-analyzed the data by taking into account the contamination of the cluster population from LMC field stars, which was completely neglected by L18a. D19 showed that $\sim 40 \%$ of the selected BSS sample (and especially the population observed along one of the two sequences) is composed of field star interlopers, concluding that the double BSS sequence is most likely a field contamination artefact.

In a recent note Li et al. (2018b, hereafter L18b) argued that the analysis by D19 is affected by two issues related to (1) the use of different HST instruments/filters in the decontamination procedure, and (2) a presumed overestimate of the number of field stars, which show a central radial segregation.

Here we demonstrate that the D19 decontamination procedure is completely unaffected by the use of different HST instruments, and that, the field stars removed by D19 have no significant radial segregation toward the cluster center. Hence, we confirm that the claimed double sequence is just a field contamination artefact.

\section{DISCUSSION}

For the field decontamination procedure, D19 made use of the Cluster and the Field Pointings, secured in the same set of filters (F475W and F814W) with the WFC3/UVIS and the ACS/WFC, respectively. Figure 1a shows that the filter systems of these two instruments are essentially the same. Indeed, Deustua \& Mack (2018) report differences smaller than $\pm 0.02 \mathrm{mag}$ (in a wide color range) between the ACS/WFC and WFC3/UVIS F814W and F475W magnitudes. Hence, for their field decontamination analysis, D19 did not "cross-calibrate the instrumental magnitudes difference between two different filter systems", at odds with what is stated by L18b. On the same line, we stress that the different main sequence (MS) slopes observed in the two HST data sets (see Figure 4 in D19) is simply due to different ages and age distributions of the cluster and field populations. The stars that survived the decontamination procedure (performed in the $m_{F 475 W}-m_{F 814 W}$ diagram), were then analysed in the $\left(m_{\mathrm{F} 814 \mathrm{~W}}, m_{\mathrm{F} 336 \mathrm{~W}}-m_{\mathrm{F} 814 \mathrm{~W}}\right)$ color-magnitude diagram (CMD - see Figure 2), where the presence of a double BSS sequence was claimed by L18a. This is straightforward since every star in the Cluster Pointing has also a measure in the F336W (see Table 1 in D19).

As for the presumed radial segregation of the D19 field contaminants, we first have to warn that the 14 objects selected by L18b as the "D19 field stars" are not the same used in D19 (see Figure 3). In Figure 1b we plot the radial 

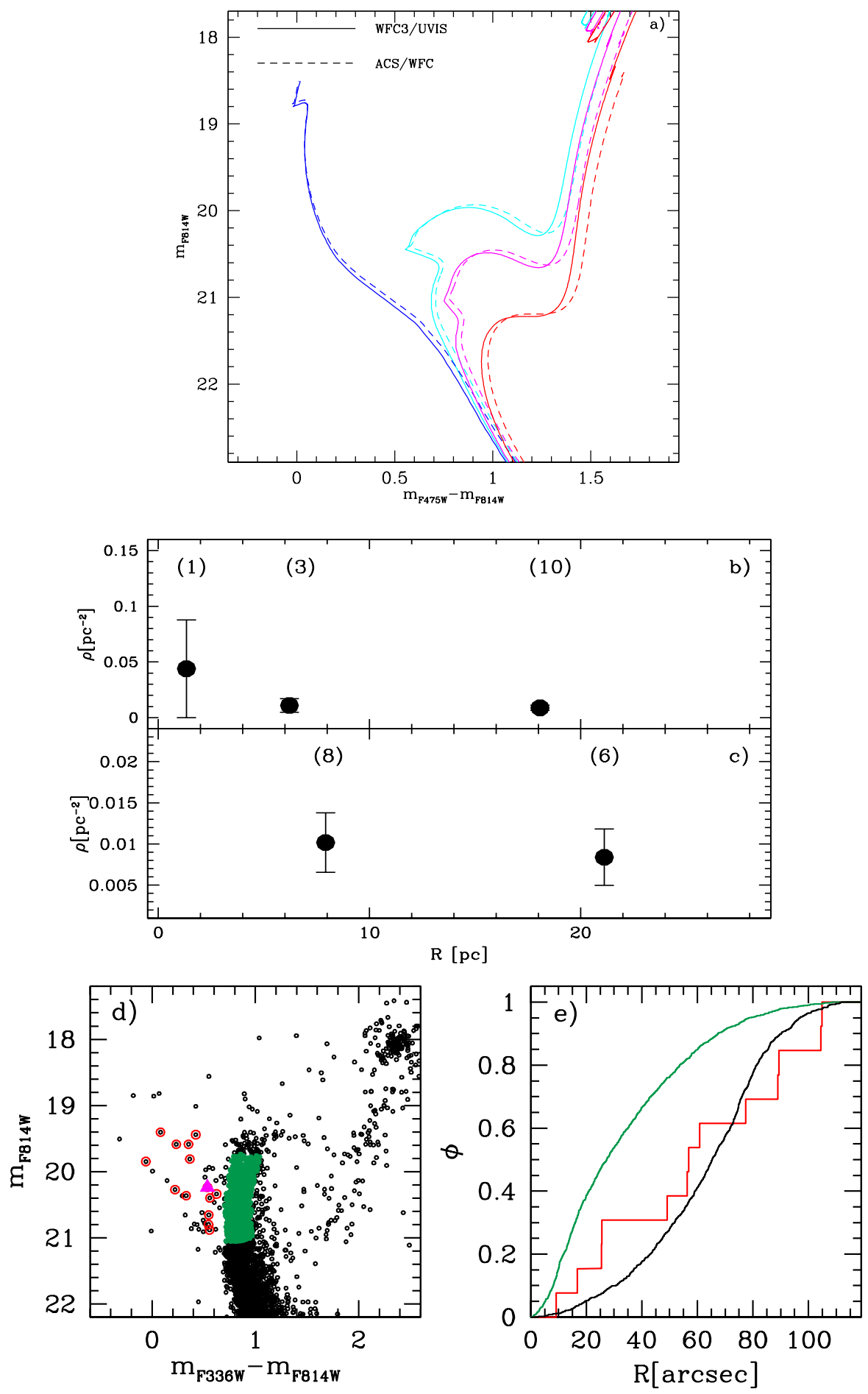

Figure 1. a): Comparison between WFC3/UVIS (solid lines) and ACS/WFC (dashed) isochrones (Pietrinferni et al. 2006) with ages of $0.4 \mathrm{Gyr}$ (blue), 2.0 Gyr (cyan), $6.0 \mathrm{Gyr}$ (magenta) and $8.0 \mathrm{Gyr}$ (red) and metallicity $[\mathrm{Fe} / \mathrm{H}] \sim-0.5$ in the $\left(m_{F 814 W}\right.$, $m_{F 475 W}-m_{F 814 W}$ ) CMD. b): Density distribution of the D19 sample of (14) removed BSSs obtained with the same radial bins adopted by Li18b and c), by dividing the field of view in two concentric radial regions covering the same area. The number of stars observed in the each radial bin is marked within brackets. d): CMD of NGC 2173 zoomed in the MS turn-off region. The red circles are the stars removed by D19 as likely field contaminants of the BSS population, the magenta triangle corresponds to the only object removed at $r<r_{c}$. Green circles mark the MS population selected as reference. e): The red and green lines show the cumulative radial distribution of the populations selected in panel d) with the same color code. The black line refers to a mock population homogeneously distributed within the WFC3 area and representative of the LMC field population. 


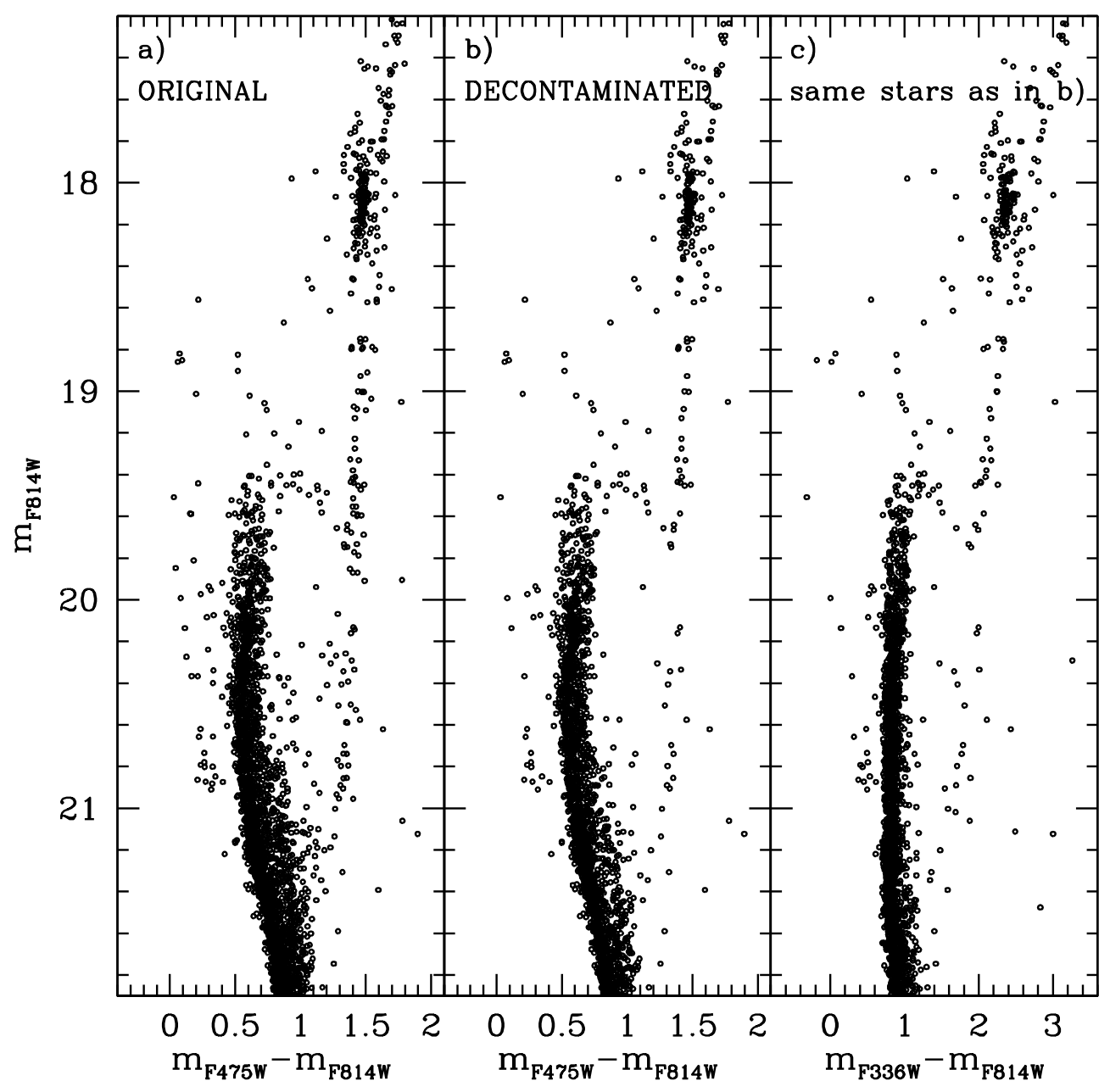

Figure 2. a): original $\left.\left(m_{\mathrm{F} 814 \mathrm{~W}}, m_{\mathrm{F} 475 \mathrm{~W}}-m_{\mathrm{F} 814 \mathrm{~W}}\right) \mathrm{CMD} ; \mathbf{b}\right)$ : CMD of stars surviving to the statistical decontamination analysis described in D19; $\mathbf{c}):\left(m_{\mathrm{F} 814 \mathrm{~W}}, m_{\mathrm{F} 336 \mathrm{~W}}-m_{\mathrm{F} 814 \mathrm{~W}}\right)$ CMD of the same stars as in panel b).

density distribution obtained by using the true D19 sample of removed stars, and the same radial bins adopted in L18b. While a quick look to the plot reveals an apparent overdensity at the center, we stress that it is caused by only one (!!) star. Very basic statistical arguments demonstrate that (because of the very poor statistics) the density is constant when Poissonian errors are taken into account: $\rho_{1}=0.044 \pm 0.044, \rho_{2}=0.011 \pm 0.006, \rho_{3}=0.009 \pm 0.002$, in units of stars $\mathrm{pc}^{-2}$. To quantify the probability that the density distribution shown in in Figure $1 \mathrm{~b}$ is obtained by chance, we run 10000 Monte Carlo simulations extracting, each time, 14 objects homogeneously distributed within the WFC3 field of view and counting the number of stars in the three adopted radial bins. We find that in more than $99.9 \%$ of the cases $1 \pm 1$ star is counted at $r<r_{c}$, thus demonstrating that the apparent central peak hasn't any statistical significance. In addition, we stress that no stars are subtracted at $r<r_{c}$ in $80 \%$ of all the decontamination runs performed in D19. We also note that if the sample is split in two concentric radial regions covering the same area, the derived BSS densities (Figure 1c) are indistinguishable $\left(\rho_{1}=0.010 \pm 0.004 \mathrm{pc}^{-2}\right.$ and $\left.\rho_{2}=0.008 \pm 0.003 \mathrm{pc}^{-2}\right)$, as expected in the case of a uniform spatial distribution, as that of the field population. The definitive demonstration that the sample of stars removed by D19 is effectively composed of likely field contaminants is shown in Figure 1e. As apparent, the cumulative radial distribution of the 14 removed stars (red circles in Figure 1d) is inconsistent (KS-test probability 0.1\%) with the cluster radial distribution (as traced by the MS stars, in green), while it is well compatible (KS-test probability 60\%) with that of a mock population of 1000 stars homogeneously distributed within the WFC3 field of view (in black), which is representative of the LMC field. 


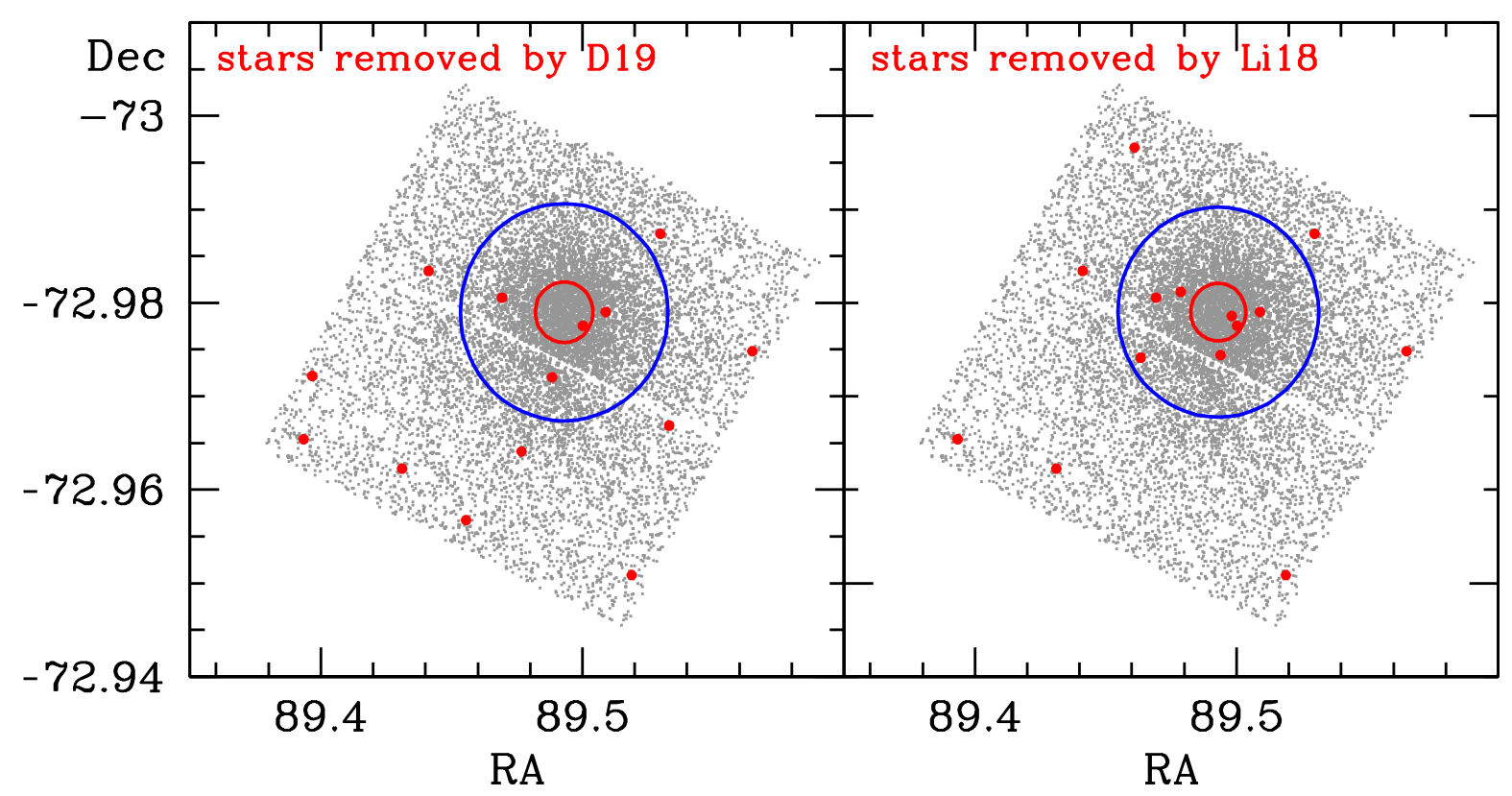

Figure 3. Comparison between the distribution of the BSS field stars removed by D19 (left panel) and those erroneously identified/selected by L18b as the "D19 removed stars" (right panel). Clearly most of them are not the same.

Clearly, these results do not change even if a $10 \%$ incompleteness is assumed within $r_{c}$ (as estimated by L18a).

As a final note, we stress that the density profile obtained from the Field Pointing remains constant with radius (see empty circles at $r>200^{\prime \prime}$ in Figure 4) as expected for field stars, thus fully justifying the use of the Field Pointing for decontamination purposes. Figure 4 shows that the structural parameters estimated by L18a well fit the observed density distribution if no subtraction of the field contribution is performed, thus causing a significant overestimate of the cluster tidal radius.

\section{CONCLUSION}

These results demonstrate that most of the 14 stars removed by D19 are indeed field interlopers. Note that even by conservatively assuming that the star removed in the central bin (magenta triangle in Figure 1d) is a cluster member, the BSS region in the CMD of NGC 2173 remains populated by only one sequence. Therefore the conclusions presented in D19 remain unchanged:

(i) The Field Pointing is perfectly suitable to estimate the field star contamination of NGC 2173.

(ii) The D19 analysis does not overestimate the BSS contamination level.

(iii) The presumed double BSS sequence in NGC 2173 is a contamination artefact, since when field contamination is properly taken into account, only a single and scarcely populated BSS sequence appears. 


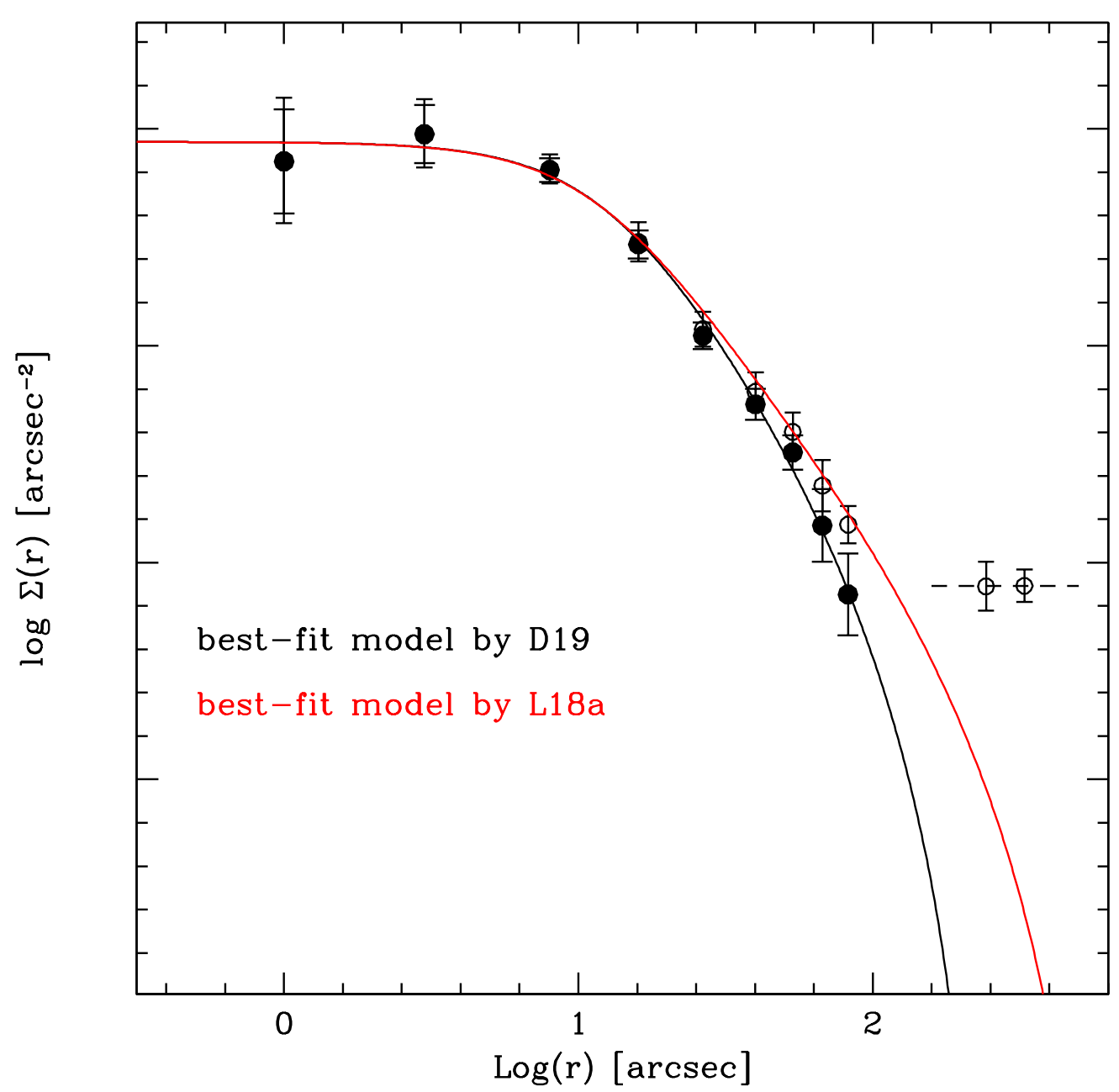

Figure 4. Observed and decontaminated star count density profile of NGC 2173 (the same as in Figure 3 of D19). The dashed line represents the density value of the background as derived by averaging the two outermost radial bins, which have been obtained from the Field Pointing. The best-fit King model obtained by D19 is shown in black, while the the model corresponding to the structural parameters quoted by L18a is shown in red. Clearly the red model fits the non-background subtracted profile while it is unable to reproduce the cluster density distribution properly corrected for the LMC field contamination.

\section{REFERENCES}

Dalessandro, E., Ferraro, F. R., Massari, D., et al. 2013, ApJ, 778, 135

Dalessandro, E., Ferraro, F. R., Bastian, N., et al. 2019, A\&A, 621, A45 (D19)

Deustua. S.E., \& Mack, J., Instrument Science Report ACS-2017-10, Instrument Science Report WFC3-2018-02

Ferraro, F. R., Beccari, G., Dalessandro, E., et al. 2009, Nature, 462, 1028
Li, C., Deng, L., de Grijs, R., Jiang, D., \& Xin, Y. 2018, ApJ, 856, 25 (L18a)

Li, C., Deng, L., de Grijs, R., Jiang, D., \& Xin, Y. 2018, Research Notes of the American Astronomical Society, 2, 215 (L18b)

Pietrinferni, A., Cassisi, S., Salaris, M., \& Castelli, F. 2006, ApJ, 642, 797

Simunovic, M., Puzia, T. H., \& Sills, A. 2014, ApJL, 795, L10 